\title{
The influence of inter-atomic transitions in Auger valence band spectroscopy: oxygen on $\mathrm{Si}(001) 2 \times 1$
}

\author{
Herbert Wormeester ${ }^{\mathrm{a}, *}$, Herman J. Borg ${ }^{\mathrm{b}}$ and Arend van Silfhout ${ }^{\mathrm{a}}$ \\ a University of Twente, Faculty of Applied Physics, P.O. Box 217, 7500 AE Enschede, Netherlands \\ ${ }^{b}$ Eindhoven University of Technology, Laboratory of Inorganic Chemistry and Catalysis, P.O. Box 513, 6500 MB Eindhoven, \\ Netherlands
}

Received 31 January 1991; accepted for publication 4 June 1991

\begin{abstract}
In this paper we will show that the description of an Auger process in terms of a process confined to one atom is in general not adequate and the Mulliken population is only in very specific cases a good alternative in evaluating the strength of inter-atomic transitions. The ionicity of the chemical bond cannot be used as a direct measure of the contribution of inter-atomic Auger transitions, as will be demonstrated in the case of the oxygen KVV Auger transitions in gaseous molecular oxygen and oxygen chemisorbed on the Si(001) surface. A full evaluation of inter-atomic transition rates shows that their strength depends on the inter-atomic distance as well as on the screening of the initial core hole.
\end{abstract}

\section{Introduction}

Since the discovery of the Auger effect [1] one has tried to calculate Auger transition rates [2-6]. The attention was mainly focused on transitions involving core electrons, in which case the Auger electron emission process can be regarded as a strong localized phenomenon and an atomic model can be used. Only electrons belonging to the atom where the initial core hole, initiator of the Auger process, is situated have to be taken into account. Over the years calculations have become highly accurate in the prediction of the Auger line shape as a function of the atomic number $Z$, and many features in addition to the simple Auger process like shake-up and shake-off and plasmon loss $[7,8]$ have been studied. The main attention was devoted to the energy position of the features and less to their intensity. An understanding of the Auger process, a many-particle problem, was given by the work of, for instance Asaad and Burhop [9] and Shirley [10].

\footnotetext{
* To whom correspondence should be addressed.
}

One has also tried to obtain information about the chemical bond in addition to mere chemical element detection by measuring the shift in energy position of relevant features. The foundation of this kind of work has been laid by Siegbahn et al. [11]. In electron spectroscopy for chemical analysis (ESCA), Siegbahn et al. [11] related the energy shift of X-ray photoemission peaks directly to the kind of chemical bonds involved. This concept, based on the ionicity of a bond, is, however, not as easy applicable in Auger electron spectroscopy (AES).

The first observation of the Auger effect in which valence electrons are directly involved was made by Lander [12]. The energy distribution of the Auger electrons recorded, now reflects the distribution of the valence electrons at the atoms where the Auger process is generated and is as such a probe of the chemical bond. Lander [12] suggested that in a first-order approximation the Auger lineshape can be seen as a self-convolution of the valence band. One of the first reports to extract valence band information from the Auger lineshape of silicon was given by Amelio [13]. He observed that the Si LVV Auger lineshape 
changes upon exposing the clean silicon surface to molecular oxygen, and tried to derive an image of the valence band for the clean and oxidized Si surface by performing a self-deconvolution of the Auger lineshape. Since this observation, Auger valence band spectroscopy has become a widely used tool in the study of the influence of adsorbates on a substrate. Nowadays AES can be regarded as a complementary tool to ultraviolet photo-electron spectroscopy (UPS) for probing the valence band. With AES it is possible to tune into the valence band electron distribution of the substrate or adsorbate atoms separately. Moreover, it is possible to determine the distribution of valence electrons with a rather high binding energy $(\sim 20 \mathrm{eV})$. In this energy region UPS is less sensitive to these structures duc to a large emission of secondary electrons. Compared with UPS, AES is one of the standard and low-cost surface analysis tools in UHV and is an easy to usc experimental technique. However, the interpretation of the features in the Auger lineshape, their energy position and intensity, is not as straightforward as in UPS. A good understanding of all phenomena associated with the Auger process is therefore important. Many-body effects associated with the two-hole final state may lead to quasi atomic lineshapes, as shown by Cini [14] and Sawatzky [15]. In the case of gaseous molecular oxygen and oxygen adsorbed on a single-crystalline surface, the material studied by us, final state correlation effects can be neglected, because the width of the oxygen valence band is larger than the effective Coulomb interaction of the two holes in the valence band. The use of the oxygen Auger lines not only has the virtue of a relatively easy to understand and calculate process from the theoretical point of view. Experimentally the oxygen Auger spectrum is also favourable as the features present can be distinguished clearly. The background subtraction in the energy region where oxygen Auger transitions occur is also less complicated than in the low kinetic energy region, e.g., SiLVV, where secondary electrons generated by the incident electron beam may inhibit a straightforward background correction [16]. In addition to the determination of the chemical composition at the sur- face and the nature of the chemical bonds involved, the angle dependence of the emitted Auger electrons can be used to obtain information on the geometric structure $[17,18]$. Forward scattering phenomena [19] have recently been added as a promising field of application.

Feibelman et al. [4,5] were the first to calculate the Auger valence band lineshapes of the $\mathrm{Si}$ surface. These lineshapes exhibited, however, a rather large deviation from the experimentally obtained Auger spectra. Jennison [20,21] concluded from this deviation, first, that transition rates for the Auger process should be calculated with the same type of wave functions as the electronic structure of the material under study has been calculated with and, second, that AES probes the local atomic charge distribution in the case of a clean Si surface. As a result of this second conclusion the s type electrons, mainly responsible for the bond, are believed not to contribute as strongly to the lineshape as would be the case in atomic silicon. However, this view of a strongly localized process conflicts with the mcasurcments on $\mathrm{Pd}_{4} \mathrm{Si}$ and $\mathrm{NO}_{3}^{-}$, in which cases this behaviour is not observed $[8,22]$ and for which has been shown that the bonding charge influences the lineshape unambiguously. The experimental Auger lineshapes resembled a Mullikenpopulation like [23] probe of the electron distribution. Ramaker $[24,25]$ introduced the final state (FS) and orthogonal final state (OFS) rules for AES to give some insight into the kind of electron distribution that has to be used for calculating the relative intensity and shape of the partial, angular momentum dependent, lineshape. However, also with these rules the discrepancy between the electron distribution measured for $\mathrm{Si}$ and $\mathrm{Pd}_{4} \mathrm{Si}$ was not resolved. It made the imputed small contribution of the s electrons to the $\mathrm{Si}$ lineshape even more peculiar, as stated by $\mathrm{Ra}$ maker [24]. The question what electron population is probed is therefore crucial for the understanding and interpretation of Auger processes involving valence electrons. Fact is that the Auger process is well understood as long as valence electrons are not involved in the Auger transition. On the other hand, the casual resemblance of the Auger lineshape with a Mulliken popula- 
tion or a local population $[8,22]$ raises the question what the influence of the valence electrons, involved in the chemical bond, is on the Auger lineshape. In order to gain an insight into this problem we must study the influence of nearestneighbour (nn) atoms on the Auger process, that is, we have to include inter-atomic Auger effects. In this paper we will show that a profound calculation of the transition rate of an electron which does not belong to the atom where the core hole is created, unambiguously indicates the importance of inter-atomic Auger transitions relative to intra-atomic effects.

\section{Strength of inter-atomic transitions}

The first attempt to evaluate the transition rate of such inter-atomic transitions was performed by Matthew and Komninos (MK) [26]. The valence electrons are in this evaluation characterized by wave functions $\phi_{\mathrm{A}_{\mathrm{y}}}$ and $\phi_{\mathrm{B}_{\mathrm{v}}}$ centered at atom $\mathrm{A}$ and $\mathrm{B}$, respectively. With $\phi_{\mathrm{A}_{\mathrm{c}}}$ the wave function of the core hole and $\phi_{\mathrm{f}}$ characterizing the Auger electron, with a summation over all states of the valence electrons $A_{v}$ and $B_{v}$, the transition rate $1 / \tau$ can be written from first-order perturbation theory [2] as:

$$
\begin{aligned}
& \frac{1}{\tau}=\frac{2 \pi}{\hbar} \sum_{\substack{\text { all } \\
\text { states }}}\left|M^{\prime}\right|^{2} \delta\left(E_{\mathrm{A}_{\mathrm{v}}}+E_{\mathrm{B}_{\mathrm{v}}}-E_{\mathrm{A}_{\mathrm{c}}}-E_{\mathrm{f}}\right) \\
& =V \frac{m c^{2} \alpha^{2}}{(4 \pi)^{2} \hbar} k \int_{\Omega_{\mathrm{k}}}\left|D\left(\mathrm{~A}_{\mathrm{v}}, \mathrm{B}_{\mathrm{v}}\right)\right|^{2} \mathrm{~d} \Omega_{k}, \\
& D\left(\mathrm{~A}_{\mathrm{v}}, \mathrm{B}_{\mathrm{v}}\right)=\iint \phi_{\Lambda_{\mathrm{c}}}^{*}\left(\boldsymbol{r}_{1}\right) \phi_{\mathrm{f}}^{*}\left(\boldsymbol{r}_{2}\right) \frac{e^{2}}{r_{12}} \phi_{\mathrm{A}_{\mathrm{v}}}\left(\boldsymbol{r}_{1}\right) \\
& \times \phi_{\mathrm{B}_{v}}\left(\boldsymbol{r}_{2}\right) \mathrm{d} \boldsymbol{r}_{1} \mathrm{~d} \boldsymbol{r}_{2},
\end{aligned}
$$

where $V$ is the detection volume, $\alpha$ the hyperfine constant and the integration is taken over the emission angle $\Omega_{k}$ of the Auger electrons with momentum $k$. As a result of the Pauli principle the direct transition term $D\left(\mathrm{~A}_{\mathrm{v}}, \mathrm{B}_{\mathrm{v}}\right)$ has its exchange counterpart $E\left(\mathrm{~B}_{\mathrm{v}}, \mathrm{A}_{\mathrm{v}}\right)$ and the matrix element $D$ is substituted by $M[7,9]$;

$M=D^{*} D+E^{*} E-\operatorname{Re}\left(D^{*} E\right)$.

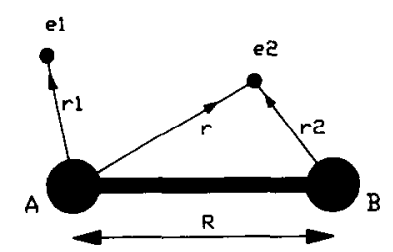

Fig. 1. Bi-atomic coordinate system for the calculation of inter-atomic Auger matrix elements involving the electrons e1 and 2 . The initial core hole is located at atom $\mathrm{A}$.

In the case of inter-atomic transitions, the nucleus $\mathrm{B}$ is at a distance $R$ from nucleus $\mathrm{A}$. A series expansion of the inter-electronic distance $r_{12}$ is made, with $z_{2}=R+z$, where $\mathrm{AB}$ is chosen as the $z$-direction (fig. 1). In contrast to MK we make this expansion in one step.

$$
\begin{aligned}
r_{12}^{2}= & R^{2}\left(1+\frac{r_{1}^{2}}{R^{2}}+\frac{r_{2}^{2}}{R^{2}}+\frac{r_{1} \cdot r_{2}}{R^{2}}\right. \\
& \left.+\frac{r_{1}}{R} \sin \vartheta_{1}+\frac{r_{2}}{R} \sin \vartheta_{2}\right) \\
= & R^{2}(1+u), \\
\frac{1}{r_{12}}= & (R \sqrt{1+u})^{-1}=\frac{1}{R}\left(1-\frac{3}{2} u+\frac{15}{8} u^{2}+\ldots\right) .
\end{aligned}
$$

With this expansion it is possible to calculate the inter-atomic transition rate as a correction on the intra-atomic transition rate. The first term in this expansion is zero because of the orthogonality of the wave functions involved. MK obtain a firstorder correction of their two-step expansion of $1 / r_{12}$ in which already a few correction terms are neglected. As the radial dependence is separated for the two electrons, these terms can be written in terms of radiative transition rates as done by MK and compared to the intra-atomic transition rates. MK therefore arrived at the conclusion that inter-atomic contributions can be neglected in the cases studied. However, as we calculated the first-order term, we obtain an OKVV interatomic transition rate that cannot be neglected in the case of an oxygen atom adsorbed on a silicon surface, a configuration discussed in section 6 . The second-order correction term is even larger than the first-order correction and therefore the 
convergence of this expansion is questionable. This implies that the expansion in eq. (3) is not valid in all cases. The conclusion of $\mathrm{MK}$ that "inter-atomic Auger matrix elements will be competitive only for very low energy Auger processes in which the intra-atomic process is very slow" cannot be sustained. The validity of the expansion of eq. (3) has to be tested in the specific configuration studied in the case of Auger valence band spectroscopy. Applying the expansion of eq. (3) for a specific geometric configuration therefore involves a test of the validity of this equation by calculating the second order correction term. The reason for the rather high contribution of the inter-atomic transitions is the final state wave function. The initial state wave functions $\phi_{A_{v}}$ and $\phi_{B_{\mathrm{V}}}$ are confined to the nucleus from which they originate and this certainly holds for the core hole wave function $\phi_{A_{c}}$. The final state wave function is not strictly confined to the nucleus from which the Auger electron is emitted (B). 'I he amplitude of this function has a value that cannot be neglected at the inter-atomic distance $R$. The radial integrals of the clectron from atom $B$ therefore need an integration range exceeding the inter-atomic distance $R$. From the above analysis it is evident that the electrons regarded to belong to the atom where the initial core hole is created cannot give a correct Auger lineshape. This raises the question whether an electron distribution like the Mulliken population, in which case the Auger process can still be treated as a pure intra-atomic process, can be used as a good approximation for the detection volume and to predict Auger lineshapes.

\section{Mulliken versus local population}

Hutson et al. [22] and Ramaker [8] have made an attempt to provide a solution for the question what charge is probed by AES. They compared the Auger transition rate including inter-atomic transitions with a local or a Mulliken population. We will give a short review of their idea and a comment. Consider the two-atom system of fig. 1, with wave function $\psi=a \phi_{\mathrm{A}}+b \phi_{\mathrm{B}}$. The Auger transition rate can then be written, with $\phi_{\mathrm{c}}$ and $\phi_{\mathrm{f}}$ the core wave function and the Auger electron wave function, respectively, as:

$$
\begin{aligned}
& I_{\exp } \sim {\left[\left\langle\phi_{\mathrm{c}} \phi_{\mathrm{f}}\left|\frac{e^{2}}{r_{12}}\right| a^{2} \phi_{\mathrm{A}}^{2}+b^{2} \phi_{\mathrm{B}}^{2}\right.\right.} \\
&\left.\left.+a b \phi_{\mathrm{A}} \phi_{\mathrm{B}}+b a \phi_{\mathrm{B}} \phi_{\mathrm{A}}\right\rangle\right]^{2} \\
& I_{\mathrm{exp}} \sim a^{4} M_{\mathrm{cAA}}^{2}+2 a^{3} b\left(M_{\mathrm{cAB}}+M_{\mathrm{cBA}}\right) M_{\mathrm{cAA}}+\ldots
\end{aligned}
$$

$M_{\text {cAA }}, M_{\text {cAB }}$ and $M_{\text {cBA }}$ denote here the Auger matrix element of the pure intra-atomic transition, the intra-inter- and the inter-intra-atomic transition, respectively. It is assumed that $M_{\mathrm{cAA}}$ $>M_{\mathrm{cAB}}, M_{\mathrm{cBA}}>M_{\mathrm{cBB}}$. The experimentally observed Auger transition rate $I_{\exp }$ is now compared with the local and Mulliken population, with $S$ the overlap integral between $\phi_{\mathrm{A}}$ and $\phi_{\mathrm{B}}$

$$
\begin{aligned}
I_{\mathrm{L}} & \sim a^{4} M_{\mathrm{CAA}}^{2}, \\
I_{\mathrm{M}} & \sim\left(a^{2}+a b S\right)^{2} M_{\mathrm{cAA}}^{2} \\
& =a^{4} M_{\mathrm{CAA}}^{2}+2 a^{3} b S M_{\mathrm{CAA}}^{2}+\ldots .
\end{aligned}
$$

The second terms of eqs. (5) and (7) are equivalent if

$$
\left(M_{\mathrm{cAB}}+M_{\mathrm{cBA}}\right) / M_{\mathrm{cAA}}=S \text {. }
$$

Hutson et al. [22] now argue that since the twocenter overlap integral is typically of the order 0.1 and, according to $\mathrm{MK}$, the transition rate ratio of eq. (8) is of the order 0.01 , the Mulliken population must overestimate the population observed with Auger. In the view of Hutson et al. [22], it is possible to observe a local or a Mulliken population, depending on the material studied. However, as we showed above the analysis of $\mathrm{MK}$ is disputable. The inter-atomic transition rates are larger than expected from their analysis and may certainly be of the order of the two-center overlap integral (eq. (8)). The analysis in section 2 of this paper has shown that total neglect of the electrons in the bond, for the situation with a local population, is quite unlikely in the case of AES recording the valence band region. The question remains why measured Auger lineshapes sometimes seem to resemble a local population 
while other lineshapes represent a Mulliken population. To our knowledge the local population is mainly reported in the case of pure silicon [8]. This is according to Jennison [20,21], because AES does not sample electrons in the chemical bond. His argument was based on the work of $\mathrm{MK}$, and using this argument it was possible to fit the theoretical calculations of the Auger line shape with the experimental lineshape reducing the s-type electrons, which have a large contribution to the bonding. The theoretical line shape was now less broad and comparable with the experimental lineshape of Houston et al. [27]. Ramaker et al. [25] stated that they "do not believe that the bonding charge concept is the sole mechanism for the reduced ss and sp components". This statement was based on the conflicting result of the FS rule that suggests a larger contribution of the s electrons, and the already mentioned contradicting measurements on other materials. A careful analysis of the experimental LVV lineshape of the clean $\mathrm{Si}(001)$ surface by Sasse et al. [28,29] showed that the kinetic energy distribution of the Auger electrons was broader than recorded by Houston et al. [27]. The main problem attacked by Sasse et al. [28] was the removal of additional structures such as plasmon loss. Agreement with the theoretical lineshape has therefore been obtained without suppressing the contribution of the s-type electrons.

From the above discussion, it seems that the Mulliken analysis gives a rather good approximation of the experimental Auger electron distribution. However, this analysis depends on the validity of eq. (8), the approximation of the ratio of the transition matrix elements by the two-center overlap integral. Upon comparing the two-enter overlap with the matrix elements, it is assumed implicitly that both quantities are real numbers. From an analysis of the matrix elements it is clear that in general this will not be the case $[7,20,30]$. Interference effects of the matrix elements which are neglected in eq. (7) may cause deviations, certainly when working at surfaces where mixing of $s$ and $p$ bands occurs [5]. When a Mulliken population is used it is assumed that the matrix elements are independent of the energy of the Auger electron. The work of Hörmandinger et al.
[6] showed that this does not necessarily need to be so, certainly not when $d$ electrons are involved. Probing a Mulliken population by AES is therefore questionable.

\section{Molecular oxygen}

Intra-atomic Auger transitions are easy to evaluate using Slater-type orbitals (STO) for the valence band wave function and the core hole wave function of eq. (1). The choice of the solid state continuum final state wave function is crucial $[4,7,30,31]$. For O KVV Auger transitions the energy of the Auger electron (about $500 \mathrm{eV}$ ) is large compared to the ionization energy of the valence electrons. According to the Born approximation the Auger electron can in this case be represented by a plane wave without a large error [32]. Inter-atomic Auger transitions, on the other hand, are not evaluated that simply. We have to deal with a multicenter integral in which the radial integration area is not separable straight on. Several approaches to solve this problem were tested by us. We already demonstrated in section 2 that an expansion of the term $1 / r_{12}$ in eq. (1) is not successful. Another approach is the expansion of the STO situated on atom B (fig. 1) towards atom $\mathrm{A}$ where the core hole is located. The expansion given by Sharma [33] for STOs at a different center, however, leads again to a series that contains diverging terms.

The problems related to multicenter integrals are well known in LCAO theory. In the modified neglect of differential overlap (MNDO) cluster method this problem is solved by a multipole approximation [34-36]. This method is applicable as long as the charge distribution can be described by a finite number of multipoles as in MNDO. The final state wave function for the Auger process, a plane wave, causes the number of multipoles to be infinite. Evaluation up to the octopole did not show a decrease of the multipole moment and suggests a bad or even diverging summation.

Following a suggestion of Boys [37] that Gaussian-type orbitals (GTO) are useful in the case of multicenter integrals, Hertlein et al. [30] devel- 
oped a calculation scheme for Auger transition rates based on GTOs. According to this scheme we have calculated Auger transition rates for the O KVV lineshape regarding molecular oxygen and atomic oxygen on a $\mathrm{Si}(001)$ surface, for which the electronic structures were taken from MNDO cluster calculations. The MNDO package, however, yields results in terms of STOs and only calculates the ground state valence band. The core hole wave function was therefore defined as a 1s-type STO orthonormalized to the oxygen $2 \mathrm{~s}$ and $2 p$ valence wave functions. To achieve GTOs we approximated every STO by 10 GTOs using a least-squares method. Intra-atomic transition rates thus calculated were found to correspond in intensity and energy position with those which are calculated with an exact STO method. This method is usually applied to calculate intra-atomic rates when only core electrons are involved [3]. The large number of GTOs necessary to fit one STO is astonishing. Stewart [38] provided a recipe to find a good representation of an STO by 3 to 5 GTOs, but we noticed that a slight deviation of the fitted STO, cspccially at the center of the atom, resulted in large errors.

In fig. 2 calculated Auger KVV lineshapes of molecular oxygen with various bond distances $R$ are shown for an Auger process in which only intra-atomic transitions (left panel) occur and for one in which inter-atomic transitions are also included (right panel). From the figure can be seen that the contribution of inter-atomic transitions is neglectable at an $\mathrm{O}-\mathrm{O}$ distance of $2 \AA$, but at the equilibrium bond distance of molecular oxygen, $1.135 \AA$, inter-atomic transitions influence the Auger lineshape significantly, especially in the high energy region $\left(\mathrm{KL}_{2,3} \mathrm{~L}_{2,3}\right)$. When $R$ is decreased, additional structure appears in the lineshape due to the splitting of degenerate levels, which is in agreement with experimental observations $[39,40]$. Although the variation of the bond distance in an oxygen molecule is highly theoretical our calculations show that AES does not probe a Mulliken type population. In a pure covalent bi-atomic bonding, for example, the distribution of the overlap charge between the atoms is independent of the distance. The Mulliken charge should therefore not change. The intra-
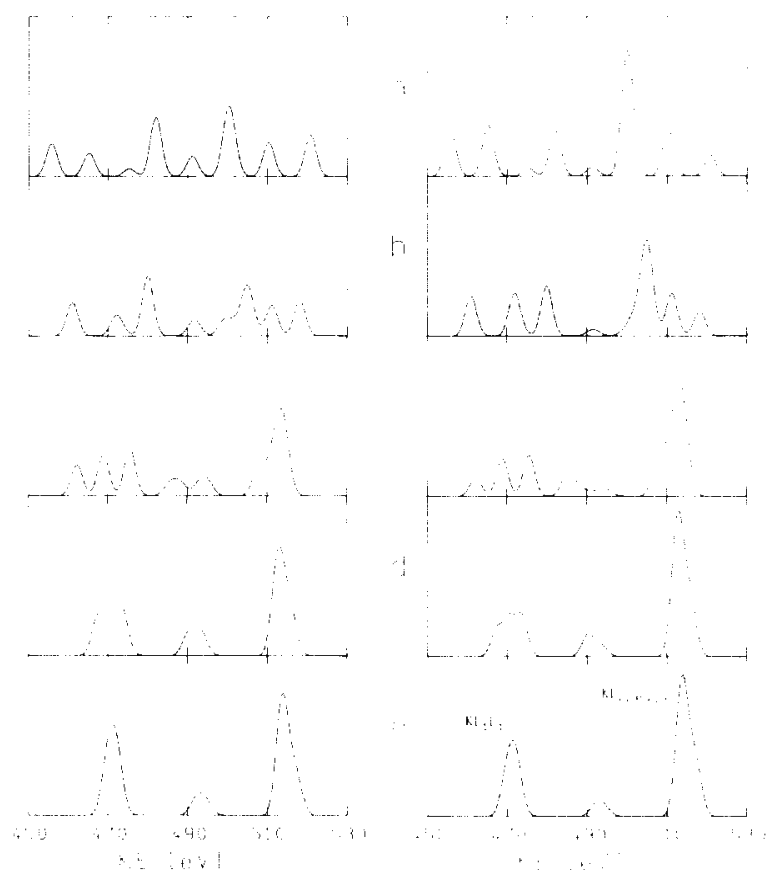

Fig. 2. OKVV Auger lineshapes of molecular oxygen at several inter-atomic distances $R$ : (a) $1.0 \AA$, (b) $1.135 \AA$ (equilibrium), (c) $1.5 \AA$. (d) $1.75 \AA$, (e) $2.0 \AA$. Ieft pant: only intra-atomic matrix elements, right panel: inter-atomic matrix elements included.

atomic matrix elements do not change either as the shape of the wave functions is not altered upon variation with $R$. This example reinforces our argument that the local population is not sampled by AES (section 3). If the electrons involved in the bond were not sampled by AES $[20,21]$, the intensities of the Auger lines would have lowered upon decreasing $R$, which is clearly not the case in fig. 2.

In the above-mentioned and following calculations we have assumed that the matrix elements are independent of energy. In fig. 3 we show the energy dependence of the intra-atomic matrix elements. As the main pp transition is concentrated at about $500 \mathrm{eV}$ and the variation of the ss matrix element is small over the energy region of interest, we can use the calculated matrix elements at $500 \mathrm{eV}$ without large error.

The density of states obtained from cluster calculations is a discrete set of electron energy levels. The calculated Auger lineshape therefore 


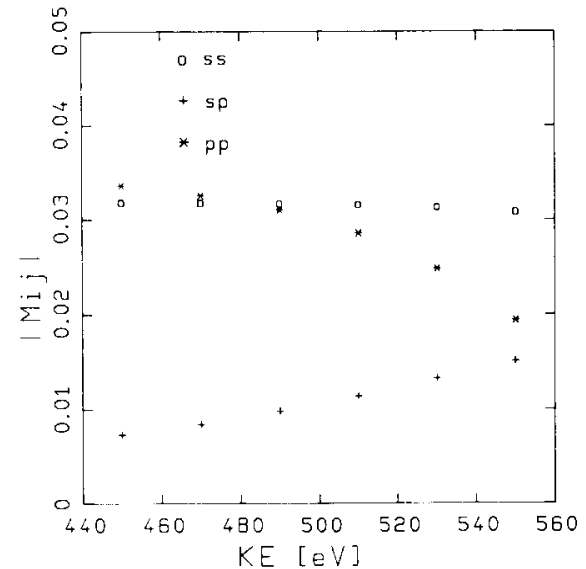

Fig. 3. Energy dependence of the ss, sp and pp intra-atomic matrix elements of the oxygen atom.

consists of discrete lines. In order to prevent this calculational artefact, the lineshape must be broadened. The transformation of discrete energy levels into continuous bands can be achieved by applying a Gaussian or Lorentzian broadening. The influence of the width of a Gaussian or a Lorentzian distribution on the OKVV lineshape was investigated. The result of this procedure is depicted in fig. 4 , in which the parameter $\alpha$ is used to characterize the shape of the OKVV transition. This parameter was introduced by Weissmann [41] as the ratio of the $\mathrm{KL}_{1} \mathrm{~L}_{1}$ and $\mathrm{KL}_{2,3} \mathrm{~L}_{2,3} \mathrm{O}$ Auger intensities and it allows for a

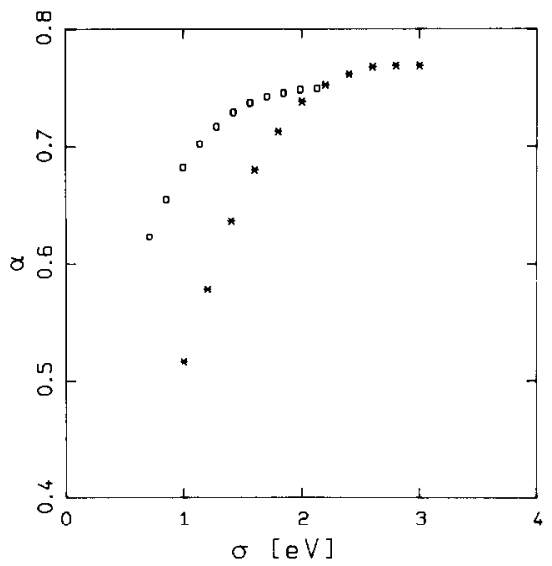

Fig. 4. Influence of the width $\sigma$ of a Gaussian (*) or Lorentzian ( $O$ ) distribution on the ratio $\alpha$ for an oxygen molecule with $R=2 \AA$. quantification of the main characteristics of the shape of the $\mathrm{O}$ Auger electrons. Under normal practical conditions, with an electron analyser operating at constant relative energy resolution, the best possible absolute resolution at about 500 $\mathrm{eV}$ is of the order of $2 \mathrm{eV}$. It is clear from fig. 4, that under such optimum conditions $\alpha$ will not be influenced by a Gaussian broadening having a width, $\sigma$, of $2 \mathrm{eV}$ or more. Selecting a smaller width, however, will lead to additional fine structure in the calculated OKVV Auger lineshapes which is due to the reappearance of the discrete energy levels. On the other hand, fine structure will arise with less progression if a Lorentzian broadening is applied (see fig. 4). But since a Lorentzian broadening is not commonly used as a broadening mechanism in AES we shall not consider its application in our calculations.

From the work of Weissmann and Müller [7] a value for the leading inter-atomic transition term relative to the intra-atomic transition term can be determined for Be. From fig. 17 of ref. [7] it can be derived that the second-order term $\left(M_{\mathrm{cAB}} /\right.$ $\left.M_{\text {cAA }}\right)^{2}=0.014$. The leading term for the interatomic contribution is however, not so small as suggested. I ooking at eq. (3.6) in ref. [7], the leading term of the inter-atomic transition rate (eq. (5) of this paper) will have a relative value of $\left(2 M_{\mathrm{cAB}} / M_{\mathrm{cAA}}\right)=0.24$. Their conclusion that inter-atomic effects do not play a role in the covalent $\mathrm{Be}$ crystal at the nearest-neighbour distance [7] can therefore not be sustained.

Auger Spectra of $\mathrm{O}_{2}$ and $\mathrm{Be}$ do show a considerable contribution of inter-atomic transitions as we have seen although both have a pure covalent bond. This implies that the ionicity of a bond is not a good measure for the occurrence of interatomic transition rates as was proposed by Weissmann [41]. Nevertheless, the ratio $\alpha$ defined by him remains a valuable tool in the study of the nature of chemical bonds. The ionicity, determined by Pauling's model of electron charges [42], is an indication of the shift of electrons from one atom to another, and therefore their probability to contribute to the Auger process. If interatomic transitions were negligible then the ionicity concept would indeed be a likely explanation for the observed difference in $\alpha$ for several ox- 
ides and carbides $[43,44]$. But as we have illustrated in our simulations of a covalent molecule (fig. 2) the bond distance, and therefore the overlap integral, is a key parameter which determines the strength of inter-atomic transitions. This aspect also explains why the Mulliken approach works quite well in many cases.

\section{Core hole screening}

The calculated oxygen Auger spectrum of $\mathrm{O}_{2}$ (see fig. 2) shows that the contribution of the s-electrons $\left(\mathrm{KL}_{1} \mathrm{~L}_{1}\right)$ relative to the p-electrons $\left(\mathrm{KL}_{2,3} \mathrm{~L}_{2,3}\right)$ is too large as compared to experimental lineshapes $[39,40]$. In terms of Weissmann's ratio $\alpha$ [41] the deviation between theory and experiment is about a factor of 10 . This discrepancy was already reported by Ramaker et al. [45]. Since in the O KVV Auger electron emission process valence electrons are involved the use of atomic matrix elements was regarded as questionable [45]. The overestimation of the intensity of the $\mathrm{KL}_{1} \mathrm{~L}_{1}$ transition with respect to the intensity of the $\mathrm{KL}_{2,3} \mathrm{~L}_{2,3}$ transition may originate from the use of a ground state electronic structure, in which the influence of the initial core hole on the distribution of the valence electrons is neglected. Ramaker introduced the FS rule [24] to describe the impact of the core hole, stating that the intensities of the components of the Auger transition, characterized by their angular momentum, are governed by the initial state in the presence of the core hole. The final state describes the waveforms of these components. However, the initial state cannot be simulated straightforward in the electronic structure calculation method used by us (MNDO), which is essentially a ground state evaluation. To obtain some insight into the difference between the excited initial state and the ground state we have studied the influence of a contraction of the wave functions, involved in the Auger process, on the OKVV lineshape, i.e., $\alpha$.

We have simulated the FS rule with what we call a frozen electron contraction. The angularmomentum dependent matrix elements were calculated with a change in the shape of the valence orbitals, while the electron distribution over these orbitals, expressed in the LCAO coefficients, was kept constant. This was achieved by changing the exponential decay range of the STOs characterizing the valence orbitals. An appropriate choice for changing the exponential decay is the substitution of the oxygen $2 s$ and $2 p$ values for those of fluorine. The fluorine value is reached at a relative contraction of $\eta=1.13$. This choice is based on the equivalent core approximation (ECA), in which a core hole is simulated by changing the charge of the nucleus from $Z$ to $Z+1[25,46]$.
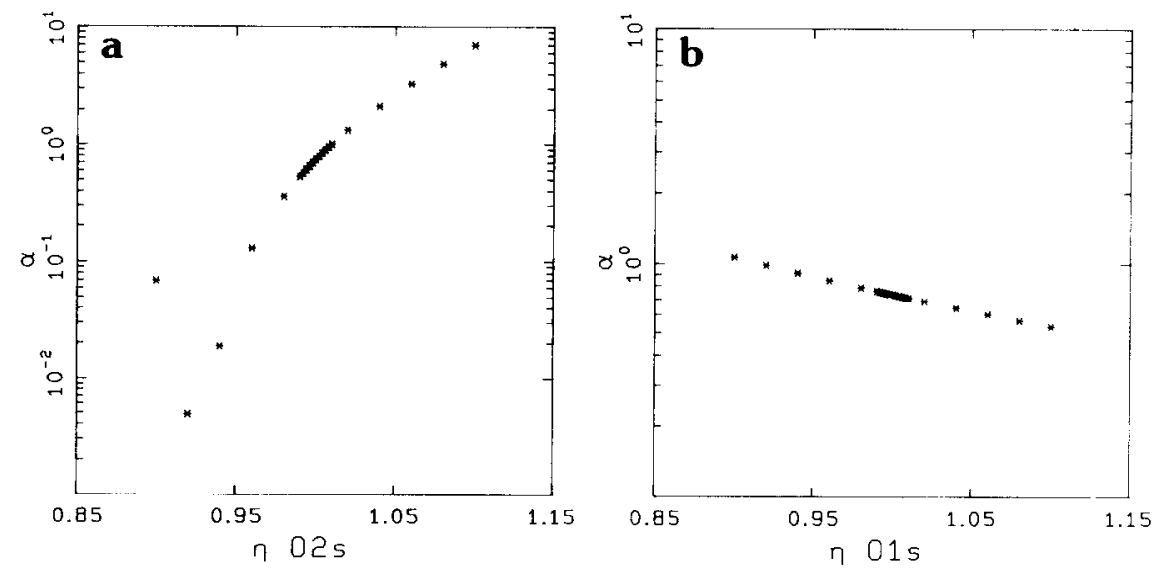

Fig. 5. (a) $\alpha$ as a function of the relative contraction of the radial exponential decay range $\eta$ for the oxygen 2 s STO. (b) as (a). for the oxygen 1s STO. 
The effect of a relative contraction $\eta$ on $\alpha$ was studied for an oxygen molecule with a bond distance of $2 \AA$. In this situation it is possible to omit the influence of inter-atomic transitions (see fig. 2). In fig. 5a the effect of a contraction of the $\mathrm{O} 2 \mathrm{~s}$ orbital on the ratio $\alpha$ is presented and it shows that $\alpha$ increases as the contraction proceeds. The discrepancy with the experimentally observed lineshape of $\mathrm{O}_{2}[39,40]$ only increases with contraction.

Until now, we did not take into account the influence of the core hole, as the wave function for this initial state cannot be evaluated in the MNDO calculation. For that purpose an ab initio method is required. The core state wavefunction was approximated by taking the exponential decay for the O1s STO from the table of Clementi and Raimondi [47] and defining it orthogonal to the $\mathrm{O} 2 \mathrm{~s}$ orbital. Fig. 5b shows that a contraction of the core hole leads to a decrease in $\alpha$, which is in better agreement with the experimental observations. A previous X-ray photo-electron spectroscopy (XPS) study has shown that the shift of the $\mathrm{C} 1 \mathrm{~s}$ core electron towards higher binding energy ( $\mathrm{BE}$ ) is accompanied by a monotonous decrease of $\alpha$ [44]. From the above discussion it can therefore be concluded that a shift of the $\mathrm{O}$ 1s state towards higher $\mathrm{BE}$ is accompanied by a contraction of its core state wavefunction, and as a result a decrease of $\alpha$.

Our results show that the ECA approach is not sophisticated enough to calculate OKVV Auger spectra as the shape of the core state wavefunction is left out in this picture. We have seen that a variation of this function can alter the results significantly. However, the influence range of inter-atomic transitions is rather independent of the core hole screening. In the remaining discussion (section 6) the effect of the geometric configuration, i.e., the bond distance, on the rate of inter-atomic transitions will be the central issue in the discussion of the behaviour of $\alpha$.

\section{Oxygen on silicon}

The possibility of characterizing various kinds of chemical bonds in compounds or adsorbates containing oxygen by means of AES was introduced by Weissmann [41]. Laudet et al. [48] monitored $\alpha$ at various stages of the oxidation of $\mathrm{Cu}$ and $\mathrm{Al}$ surfaces. From this work it is clear that during the adsorption process the intensity ratio $\alpha$ changes. The question now is what $\alpha$ causes to change. Weissmann [41] related the variation of $\alpha$ to a change in charge transfer. Grunthaner et al. [49] showed that the charge transfer in the $\mathrm{Si}-\mathrm{O}$ bond depends sensitively on the local bond geometry, so that therefore a relation between geometry and $\alpha$ is expected. As we illustrated in the preceding discussion (sections 4 and 5) a variation of the geometric structure of the adsorbate and substrate will also influence the strength of inter-atomic Auger transitions remarkably.

We investigated the behaviour of $\alpha$ in the initial adsorption stages of $\mathrm{O}_{2}$ and $\mathrm{N}_{2} \mathrm{O}$ on the Si $(001) 2 \times 1$ surface [50,51]. The results of the adsorption experiments [50] as shown in fig. 6 reveal that $\alpha$ changes significantly. In ref. [50] we already outlined that the behaviour of $\alpha$ cannot be explained by the charge transfer concept [41] as this would yield a total charge transfer of at least two electrons which is physically unlikely. The presumption that the variation in $\alpha$ can be ascribed to changes in adsorption geometry of the adsorbed oxygen surface species is based on high-resolution electron energy loss spectroscopy

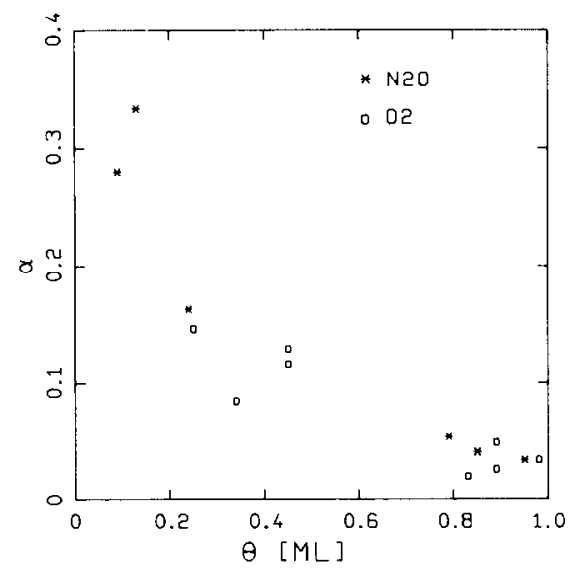

Fig. 6. The OKVV Auger intensity ratio a as a function of the fractional oxygen coverage $\theta$ upon exposing the $\operatorname{Si}(001) 2 \times 1$ surface at $300 \mathrm{~K}$ to $\mathrm{N}_{2} \mathrm{O}(*)$ and $\mathrm{O}_{2}(\mathrm{O})$ data taken from ref. [50]. 
(HREELS) work by Schaefer and Göpel [52], who observed a change in one of the frequency modes during the initial adsorption stage of molecular oxygen on the Si surface between 0.2 and 0.6 monolayer (ML) oxygen coverage, a region in which the adsorption characteristics of $\mathrm{O}_{2}$ is comparable to $\mathrm{N}_{2} \mathrm{O}[57,60]$. They related this observed change to an increase of the $\mathrm{Si}-\mathrm{O}-\mathrm{Si}$ bondangle. Their conclusion is supported by theoretical studies. In the very initial stage, the dimer bridge site has been found to be the most favourable chemisorption site as a result of theoretical work by several groups [53-59]. From the work of Zheng and Smith [57] and Miyamoto et al. $[58,59]$ it can also be seen that up to half a monolayer oxygen coverage the in-plane position of the oxygen atom $\left(R_{\perp}=0\right)$ is the most stable configuration, an adsorption geometry in which the $\mathrm{Si}-\mathrm{O}$ distance is shortest. Both calculations also show that a metastable adsorption state may exist with a relatively high value of $R_{\perp}$. This suggests that in the early adsorption stage the oxygen atom may take in its stable bridge-bonded position dynamically in which $R_{\perp}$ decreases continuously with coverage. Experimentally the bridge-bonded oxygen configuration at the dimer site was resolved as the most probable adsorption site by the work of Keim et al. [60], Schaeffer and Göpel [52] and Incoccia et al. [61].

To investigate the effect of the adsorption gcomctry on $\alpha$ we have performed a set of MNDO calculations using a $\mathrm{Si}_{9} \mathrm{H}_{12} \mathrm{O}$ cluster. Fig. 7 shows the most relevant part of this cluster, the adsorbed dimer site. In these calculations the position of the oxygen atom was varied with $R_{\perp}$, the vertical distance of the adsorbed oxygen atom relative to the adjacent $\mathrm{Si}$ atoms of the original dimer to which it is bridge bonded. In this proce-

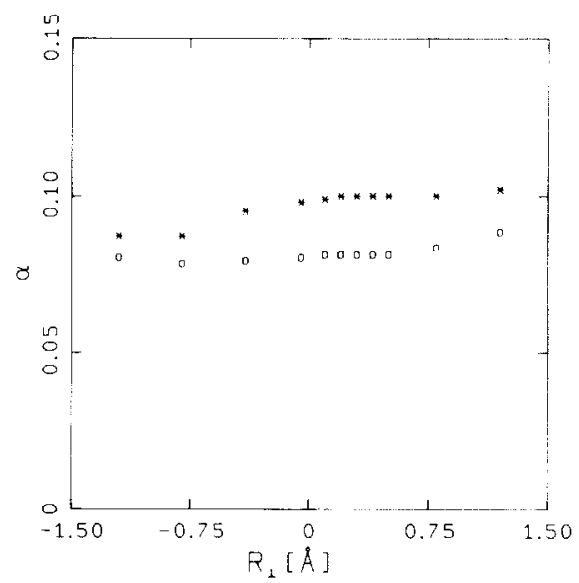

Fig. 8. The calculated ratio $\alpha$ as a function of $R$. of the bridge-bonded oxygen atom on the $\mathrm{Si}(001) 2 \times 1$ surface (see fig. 7), using the charge transfer concept of Weissmann [41] for both a local charge population (*) and a Mulliken population $(0)$.

dure the dimer tilt angle was kept constant at $12^{\circ}$ [53], and the positions of all the subsurface atoms have been kept invariant. Fig. 8 shows the result of our calculations; it shows $\alpha$ as a function of $R_{\perp}$ (fig. 7) for both a Mulliken (o) and local (*) population following the charge transfer concept of Weissmann [41]. From fig. 8 it is clear that despite a large variation in $R$, the charge transfer for both a local or a Mulliken population hardly changes, a result which shows that the concept of Weissmann [41] is not able to explain the experimentally observed behaviour of $\alpha$ (fig. 6).

The influence of $R_{\perp}$ on the Auger OKVV lineshape, especially on the strength of interatomic transitions has been evaluated using the GTO method of Hertlein et al. [30] as outlined in section 4. For comparison the lineshape for a

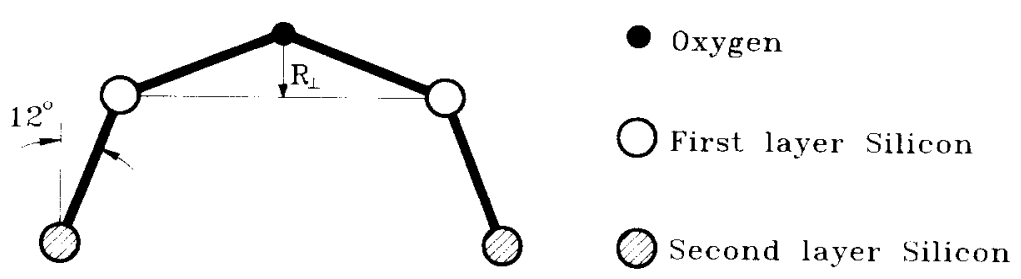

Fig. 7. Adsorption geometry of a bridge-bonded oxygen atom at the dimer site of the Si(001) $2 \times 1$ surface. In the calculations using a $\mathrm{Si}_{4} \mathrm{H}_{12} \mathrm{O}$ cluster the vertical distance $R_{1 .}$ is varied. 
pure intra-atomic Auger process was also calculated and the results are depicted in fig. 9. Upon comparing the left and right panel of this figure it can be seen that the main transition of the Auger lineshape $\left(\mathrm{KL}_{2.3} \mathrm{~L}_{2.3}\right)$ is strongly affected by inter-atomic effects as $R_{\perp}$ is varied. This result demonstrates clearly our argument that the ionicity of a bond cannot solely account for the strength of Auger transitions. Fig. 10 shows $\alpha$ as a function of $R_{\perp}$ derived from the OKVV lineshapes in the right-hand panel of fig. 9. Regarding the Auger process as a strongly localized phenomenom is not adequate to describe valence band spectroscopy. From fig. 9 a model for the early adsorption stage of oxygen on $\mathrm{Si}(001)$ can be derived in terms of a change of the bond distance. At a coverage of $\sim 0.1 \mathrm{ML}$ oxygen it is reasonable to assume that the oxygen atom is at a rather high position above the Si surface [57-59].
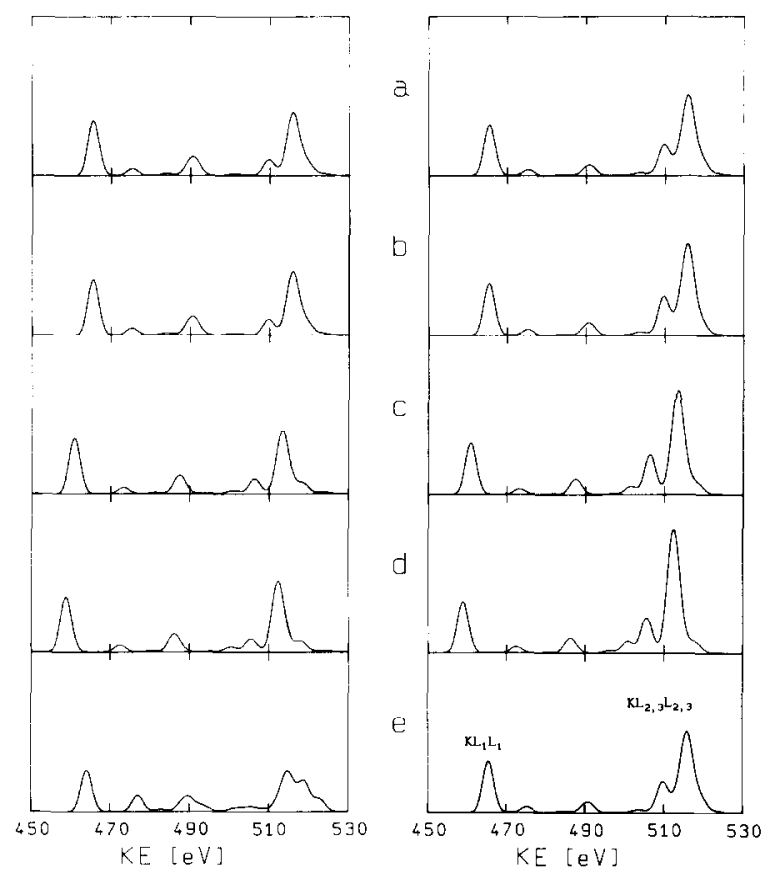

Fig. 9. OKVV Auger lineshapes for bridge-bonded oxygen at several heigths $R_{\perp}$ above the Si surface (see fig. 7): (a) $1.2 \AA$, (b) $0.8 \AA$, (c) $0.4 \AA$, (d) $0.1 \AA$, (e) $-1.2 \AA$. Left panel: only intra-atomic transitions are considered, right panel: interatomic transitions are included.

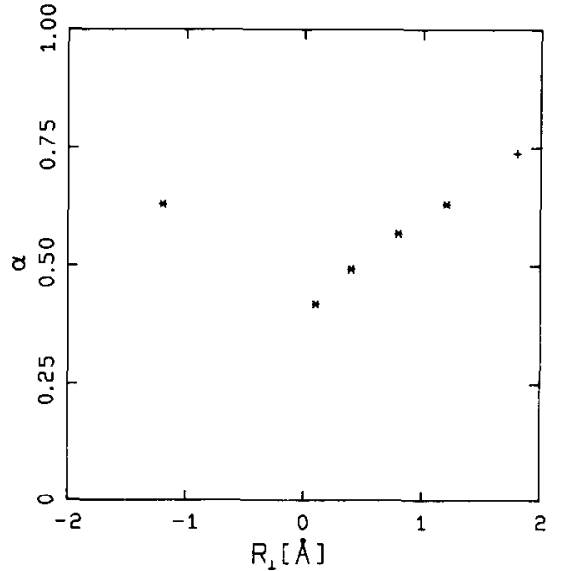

Fig. 10. Calculated intensity ratio $\alpha$ for bridge bonded oxygen on the Si surface as a function of $R_{\perp}$, derived from the right panel of fig. $9(*)$. The ratio for the free atom is achieved at infinite distance, and is derived from fig. 2 bottom $(+)$.

As the oxygen coverage increases the distance $R_{\perp}$ (see fig. 7) will decrease. The change in $R_{\perp}$ is consistent with the relaxation of the $\mathrm{Si}-\mathrm{O}-\mathrm{Si}$ bond angle as observed with HREELS [52]. This dynamic adsorption behaviour at the dimer site will also affect the adsorption energy. Gupta et al. [62] already indicated for the adsorption of molecular oxygen on the $\operatorname{Si}(111) 7 \times 7$ surface that the adsorption energy changes with coverage. A change in $\alpha$ was also observed at this surface [63], suggesting that also on this surface the Si-O bond will change as the oxygen coverage increases.

The position of the oxygen atom and its influence on the strength of inter-atomic transitions is not the only effect that has to be taken into account. The shape of the $\mathrm{O}$ core wave function is also an important factor. From ESCA studies it is well known that the $\mathrm{BE}$ of the $\mathrm{O} 1 \mathrm{~s}$ electron increases as the adsorption of molecular [52] or atomic oxygen [64] proceeds. This shift in BE has been related to a change in charge transfer which, according to Grunthaner et al. [49] can be the result of a change in bonding geometry. Also dielectric screening has been given as an explanation, although the model used by Browning et al. [65] cannot be applied satisfactorily to oxide layers thinner than $10 \AA$. From section 5 it is ex- 
pected that the observed shift in $\mathrm{BE}$ of the $\mathrm{O}$ ls state will also decrease $\alpha$.

\section{Conclusion}

We have provided evidence that the detection volume of Auger electron spectroscopy probing the valence band region is not confined to the atom where the core hole is generated, as interatomic matrix elements may show up as a significant contribution to intra-atomic matrix elements. The treatment of inter-atomic transitions as a correction term can lead to a diverging series of correction values, a result which is in contrast to the general conclusion of Matthew and Komninos [26]. Our results indicate that the local electron population cannot represent the Auger detection volume. We have also shown that the Mulliken population, although it gives for some materials good agreement between the experimental and theoretical lineshape, is in general not a good measure for the population detected as in eq. (8) two quantities are compared with a very different background. The failure of the Mulliken approach is most profound in the case of a covalent bonding, for which it is usually assumed that electrons participating in the bond do not contribute to inter-atomic Auger processes. The chemical bond ionicity concept [41], however, is unsuited to determine the strength of the inter-atomic transitions. The bond distance, in contrast, is the key parameter.

The OKVV Auger intensity ratio $\alpha$ introduced by Weissmann [41] was found to depend on the oxygen coverage at the Si(001) surface [50]. We have tried to relate the variation in $\alpha$ to a gradually changing bridge position of the oxygen atom as suggested earlier by HREELS measurcments [52]. The MNDO calculations of the O KVV Auger lineshapes for $\mathrm{O}$ on Si, and therefore $\alpha$, show that inter-atomic transitions play an important role in the behaviour of $\alpha$. A good description of the core state in the calculation is crucial in determining the transition strength of the s-electrons, and a change of this core state, as indicated by XPS measurements can also influence the Auger spectrum.

\section{Acknowledgement}

The authors are pleased to thank E.G. Keim for helpful discussions and careful reading of the manuscript.

\section{References}

[1] P. Auger, Compt. Rend. (Paris) 177 (1923) 169.

[2] G. Wentzel, Z. Phys. 43 (1927) 524.

[3] D.L. Walters and C.P. Bhalla, Phys. Rev. $\Lambda 3$ (1971) 1919.

[4] P.J. Feibelman, E.J. McGuire and K.C. Pandey. Phys. Rev. B 15 (1977) 2202.

[5] P.J. Feibelman and E.J. McGuire, Phys. Rev. B 17 (1978) 690.

[6] G. Hörmandinger, P. Weinberger and J. Redinger, Phys. Rev. B 40 (1989) 7989.

[7] R. Weissmann and K. Müller, Surf. Sci. Rept. 1 (1981) 251.

[8] D.E. Ramaker, Chemistry and Physics of Solid Surfaces IV, Vol. 20 of Springer Series in Chemical Physics (Springer, Berlin, 1982).

[9] W.N. Asaad and E.H.S. Burhop, Proc. Phys. Soc. 72 (1958) 369

[10] D.A. Shirley, Phys. Rev. A 7 (1973) 1520.

[11] K. Siegbahn, C. Nordling, A. Fahlmann, R. Nordherg, K. Hamrin, J. Hedman, G. Johanssen, T. Bergmark, S.E. Karlson, 1. Lindgren and B. Lindberg, in: ESCA-Atomic, Molecular and Solid State Structure by Means of Electron Spectroscopy, Nova Acta Regiae Societaties Scientarum, 1967, Uppsala, Ser. IV, Vol. 20.

[12] J.J. Lander, Phys. Rev. 91 (1953) 1382

[13] G.F. Amelio, Surf. Sci. 22 (1970) 301.

[14] M. Cini, Phys. Rev. B 17 (1978) 2788.

[15] G.A. Sawatzky, Phys. Rev. Lett. 39 (1977) 504.

[16] A.G.B.M. Sasse, H. Wormeester and A. van Silfhout, Surf. Interface Anal. 13 (1988) 228, and references therein.

[17] D. Aberdam, R. Baudoing, E. Blanc and C. Gaubert, Surf. Sci. 71 (1978) 279.

[18] G. Allié, E. Blanc and D. Dufayard, Surf. Sci. 57 (1976) 293.

[19] W.F. Egelhoff, J. Vac. Sci. Technol. A 4 (1986) 758.

[20] D.R. Jennison, Phys. Rev. Lett. 40 (1978) 807.

[21] D.R. Jennison, Phys, Rev. B 18 (1978) 6865.

[22] F.L. Hutson, D.E Ramaker, B.I. Dunlap, J.D. Ganjei and J.S. Murday, J. Chem. Phys. 23 (1982) 2181.

[23] R.S. Mulliken, J. Chem. Phys. 23 (1955) 1833.

[24] D.E. Ramaker, Phys. Rev. B 25 (1982) 7341.

[25] D.E. Ramaker, F.L. Hutson, N.H. Turner and W.N. Mei. Phys. Rev, B 33 (1986) 2574.

[26] J.A.D. Matthew and Y. Komninos, Surf. Sci. 53 (1975) 716. 
[27] J.E. Houston, G. Moore and M.G. Lagally, Solid State Commun. 21 (1977) 879.

[28] A.G.B.M. Sasse, H. Wormeester, M.A. van der Hoef and A. van Silfhout, Surf. Sci. 218 (1989) 553.

[29] A.G.B.M. Sasse, M.A. van der Hoef, H. Wormeester and A. van Silfhout, Solid State Commun. 71 (1989) 65.

[30] R. Hertlein, R. Weissmann and K. Müller, Surf. Sci. 77 (1978) 118.

[31] A. Liebsch, Lecture Notes for NATO Advanced Study Institute, Electron and Ion Spectroscopy of Solids, Gent, 1977.

[32] H.A. Bethe and E.E. Salpeter, Quantum Mechanics of One- and Two-Electron Atoms (Plenum, New York, 1977).

[33] R.R. Sharma, Phys. Rev. A 13 (1976) 517.

[34] K. Ohno, Theor. Chim. Acta. 2 (1964) 219.

[35] G. Klopman, J. Am. Chem. Soc. 87 (1965) 3300.

[36] M.J.S. Dewar and W. Thiel, J. Am. Chem. Soc. 99 (1977) 4899.

[37] S.F. Boys, Proc. R. Soc. A 200 (1950) 542.

[38] R.F. Stewart, J. Chem. Phys. 50 (1969) 2485.

[39] W.E. Moddeman, T.E. Carlson, M.O. Krause, B.P. Pullen, W.E. Bull and G.K. Schweitzer, J. Chem. Phys. 55 (1971) 2371.

[40] K. Siegbahn, C. Nordling, G. Johanssen, J. Hedman, P.F. Heden, K. Hamrin, V. Gelius, T. Bergmark, L.O. Werme, R. Mann and Y. Baer, ESCA Applied to free Molecules (North-Holland, Amsterdam, 1971).

[41] R. Weissmann, Solid State Commun. 31 (1979) 347.

[42] L. Pauling, The Nature of the Chemical Bond, 3rd ed. (Cornell University Press, New York, 1960).

[43] P. Ascarelli and G. Moretti, Surf. Interface Anal. 7 (1985) 8.

[44] I.M. Shul'Ga and G.I. Gutsev, J. Flectron Spectrosc. Relat. Phenom. 34 (1984) 39.

[45] D.E. Ramaker, J.S. Murday, N.H. Turner, G. Moore, M.G. Lagally and J. Houston, Phys. Rcv. B 19 (1979) 5375 .
[46] W.L. Jolly and D.N. Hendrickson, J. Am. Chem. Soc. 92 (1970) 1863.

[47] E. Clementi and D.L. Raimondi, J. Chem. Phys. 38 (1963) 2686.

[48] A. Laudet, M. Jardinier-Offergeld and F. Bouillon, Surf. Interface Anal. 8 (1986) 83.

[49] F.J. Grunthaner, P.J. Grunthaner, R.P. Vasquez, B.F. Lewis and J. Maserjian, Phys. Rev. Lett. 43 (1979) 1683.

[50] H. Wormeester, H.J. Borg, D. Terpstra, E.G. Keim and A. van Silfhout, Solid State Commun. 77 (1991) 239.

[51] E.G. Keim and A. van Silfhout, Surf. Sci. 216 (1989) L337.

[52] J.A. Schaefer and W. Göpel, Surf. Sci. 155 (1985) 535.

[53] P.V. Smith and A. Wander, Surf. Sci. 219 (1989) 77.

[54] I.P. Batra, P.S. Bagus and K. Hermann, Phys. Rev. Lett. 52 (1984) 384.

[55] I.P. Batra, P.S. Bagus and K. Hermann, J, Vac. Sci. Technol. A 2 (1984) 1075.

[56] V. Barone, F. Lelj, N. Russo and M. Toscano, Surf. Sci. 162 (1985) 230.

[57] X.M. Zheng and P.V. Smith, Surf. Sci. 219 (1990) 6.

[58] Y. Miyamoto, A. Oshiyama and A. Ishitani, Solid State Commun. 74 (1990) 343.

[59] Y. Miyamoto and A. Oshiyama, Phys. Rev. B 41 (1990) 12680.

[60] E.G. Keim, L. Wolterbeek and A. van Silfhout, Surf. Sci. 180 (1987) 565.

[61] L. Incoccia, A. Balerna, S. Cramm, C. Kunz, F. Senf and I. Storjohann, Surf. Sci. 188/190 (1987) 453.

[62] P. Gupta, C.H. Mak, P.A. Coon and S.M. George, Phys. Rev. B 40 (1989) 7739.

[63] E.G. Keim and H. Wormeester, to be published.

[64] A. Namiki, K. Tanimoto, T. Nakamura, N. Ohtake and T. Suzaki, Surf. Sci. 222 (1989) 530.

[65] R. Browning, M.A. Sobolewski and C.R. Helms, Phys. Rev. B 38 (1988) 13407. 